\title{
NORMAL FORMS FOR UNARY PROBABILISTIC AUTOMATA
}

\author{
Maria Paola Bianchi ${ }^{1}$ and Giovanni Pighizzini ${ }^{1}$
}

\begin{abstract}
We investigate the possibility of extending Chrobak normal form to the probabilistic case. While in the nondeterministic case a unary automaton can be simulated by an automaton in Chrobak normal form without increasing the number of the states in the cycles, we show that in the probabilistic case the simulation is not possible by keeping the same number of ergodic states. This negative result is proved by considering the natural extension to the probabilistic case of Chrobak normal form, obtained by replacing nondeterministic choices with probabilistic choices. We then propose a different kind of normal form, namely, cyclic normal form, which does not suffer from the same problem: we prove that each unary probabilistic automaton can be simulated by a probabilistic automaton in cyclic normal form, with at most the same number of ergodic states. In the nondeterministic case there are trivial simulations between Chrobak normal form and cyclic normal form, preserving the total number of states in the automata and in their cycles.
\end{abstract}

Mathematics Subject Classification. 68Q45, 68Q10.

\section{INTRODUCTION}

It is well-known that the transition graph of every deterministic finite automaton (DFA) over a one-letter input alphabet (a unary automaton) consists of an initial path, followed by a cycle.

\footnotetext{
Keywords and phrases. Unary languages, normal form, probabilistic automata.

1 Dipartimento di Informatica, Università degli Studi di Milano, Via Comelico 39, 20135 Milano, Italy. bianchi@di.unimi.it

pighizzini@di.unimi.it
} 
In 1986, Chrobak [2] obtained a normal form for unary nondeterministic finite automata (NFA). The transition graph of an automaton in this form consists of an initial path, as in the deterministic case, and of a set of disjoint deterministic cycles, instead of a single cycle. Each cycle can be reached from the last state of the path via a unique connecting edge. Hence, in this restricted form, the only nondeterministic choice is taken after the automaton reaches the last state of the path. Chrobak proved that each $n$-state unary NFA can be converted into an equivalent automaton in this form with at most $n$ states in the cycles and $O\left(n^{2}\right)$ states in the initial path. (For precise bounds for the number of states in the initial path and in the cycles, see [3]. In [14] a flaw in Chrobak's proof has been discussed and fixed).

Chrobak normal form turned out to be a fundamental tool in the investigation of properties of unary automata, in particular for studying unary automata simulations (see, e.g., [8]). Hence, it is natural to investigate whether or not it can be extended to other families of unary devices, not having a deterministic behavior.

In this paper we focus our attention on probabilistic finite automata (PFAs) accepting unary languages with an isolated cut-point. Informally, in these devices the transition function is replaced by a probabilistic distribution. The accepted language is defined as the set of words whose acceptance probabilities exceed a fixed threshold (the cut-point). It is also required that there are no words accepted with a probability arbitrarily close to the threshold (i.e., the cut-point must be isolated). A fundamental result by Rabin [12] states that PFAs with isolated cutpoints accept only regular languages. (Throughout the paper, we will consider only isolated cut-points).

In [10], the authors observed that for each integer $N$ there exists a language accepted by a unary 2-state PFA such that any equivalent DFA requires at least $N$ states. This means that it is impossible to get an upper bound on the number of states needed to simulate unary PFAs by equivalent DFAs or NFAs. Actually, this unbounded number of states is in the initial path of the resulting DFA, i.e., it is related to the "nonperiodic" part of the language. For the cyclic part the situation is different: in the same paper the authors proved that each unary $n$-state PFA can be simulated by a DFA with $\mathrm{e}^{O(\sqrt{n \cdot \ln n})}$ states in its cycle. This is exactly the tight upper bound for the number of the states of a DFA simulating a given unary $n$-state NFA obtained in [2]. This result stimulated further investigations, with the aim of comparing the sizes of unary DFAs, NFAs, and PFAs, in particular considering cyclic languages or the periodic parts of regular languages (see, e.g., $[1,4,9]$ ).

In this work, we give a closer look to the structure of unary PFAs. In particular, we want to investigate the possibility of extending Chrobak normal form to the probabilistic case. In other words, we are wondering whether or not given a unary $n$-state PFA it is possible to get an equivalent PFA, without significantly increasing the number of states, where probabilistic decisions can be taken only in one state and at most one time during each computation. It is not difficult to prove that this cannot be done, due to the cost of the "nonperiodic" part of the language, that can be unbounded. On the other hand, the above mentioned simulation result 
from [10] suggests the idea of restricting the attention to unary cyclic languages: it is known that for these languages turning a NFA in Chrobak normal form does not increase the total number of states. In the paper, we prove that the same does not hold in the probabilistic case. In fact we show the existence of a unary cyclic language accepted by a PFA with less states than any equivalent PFA in Chrobak normal form (the natural extension of the Chrobak normal form for NFAs). We then propose a different kind of normal form, called cyclic normal form. In this form, a PFA is a collection of disjoint deterministic cycles, each containing exactly one final state. During a computation, the only nondeterministic decision is taken at the beginning, to select the initial state according to a probabilistic distribution. Our main result shows that each $n$-state unary PFA accepting a cyclic language can be converted into a unary PFA in cyclic form without increasing the number of states. As a consequence, a PFA in cyclic form can be smaller than any equivalent PFA in Chrobak normal form. In the case of nondeterministic devices, the two forms are closely related (even dropping the restriction to the cyclic case): each NFA in Chrobak normal form can be easily converted into the nondeterministic counterpart of cyclic form and vice versa, preserving, in both transformations, the number of states.

Finally, we discuss the natural extension of the cyclic normal form to all unary regular languages.

\section{Preliminaries}

For a set $S, S^{c}$ denotes its complement and $|S|$ its cardinality. The set of natural numbers is denoted by $\mathbb{N}$, while the set of real numbers by $\mathbb{R}$. By the Fundamental Theorem of Arithmetic, any $d \in \mathbb{N} \backslash\{0\}$ admits a factorization as $d=p_{1}^{k_{1}} p_{2}^{k_{2}} \cdots p_{n}^{k_{n}}$, for primes $p_{1}, p_{2}, \ldots, p_{n}$ and $k_{1}, k_{2}, \ldots, k_{n} \in \mathbb{N} \backslash\{0\}$, which is unique except for the order in which the primes occur. Given a vector $x \in \mathbb{R}^{n},(x)_{i}$ denotes the $i$ th component of $x$ and $|\cdot|$ is the modulus function. We denote by $e_{j}$ the boolean row vector such that $\left(e_{j}\right)_{i}=1 \Leftrightarrow i=j$. For vectors $x \in \mathbb{R}^{n}$ and $y \in \mathbb{R}^{m}$, the direct sum of $x$ and $y$ is the vector $x \oplus y \in \mathbb{R}^{n+m}$ whose first $n$ components coincide with $x$ and last $m$ components with $y$. For a matrix $A \in \mathbb{R}^{n \times m}$, we denote by $(A)_{i, j}$ its $(i, j)$ th entry. A square matrix in $\mathbb{R}^{n \times n}$ is said to be of order $n$. A null matrix is denoted by 0 . For matrices $A \in \mathbb{R}^{n \times m}$ and $B \in \mathbb{R}^{p \times q}$, their direct sum is the $(n+p) \times(m+q)$ block diagonal matrix $A \oplus B=\left[\begin{array}{cc}A & 0 \\ 0 & B\end{array}\right]$.

A real matrix $M$ is said to be (sub)stochastic whenever its entries are from the interval $[0,1]$, and each row sum is (less than or) equal to 1 .

Some useful definitions and properties concerning square stochastic matrices (see, e.g., $[5,13])$ are quickly recalled hereafter.

Definition 2.1. A set $S$ of indices for a stochastic matrix $M$ is called an ergodic class if and only if for every $i, j \in S$ there exists $h \in \mathbb{N}$ such that $\left(M^{h}\right)_{i, j}>0$, and for every $i, j$ such that $i \in S$ and $j \notin S$ it holds $(M)_{i, j}=0$. 
Definition 2.2. A stochastic matrix $M$ is said to be in canonical form if and only if the indices of the same ergodic class form a set of consecutive integers, and they are smaller than the indices which do not belong to any ergodic class.

By Definition 2.2, a stochastic matrix $M$ in canonical form with $m$ ergodic classes has the following structure:

$$
M=\left[\begin{array}{ccccc}
M_{1} & 0 & \ldots & 0 & 0 \\
\vdots & & \ddots & & \vdots \\
0 & 0 & \ldots & M_{m} & 0 \\
T_{1} & T_{2} & \ldots & T_{m} & T_{m+1}
\end{array}\right]
$$

where, for $1 \leq j \leq m, M_{j}$ is the stochastic matrix representing the $j$ th ergodic class while, for $1 \leq j \leq m+1, T_{j}$ is a substochastic matrix. It is well-know [5,13] that, by performing a suitable index permutation, any stochastic matrix can be put in canonical form. Thus, without loss of generality, we may assume our stochastic matrices to be in canonical form.

Theorem 2.3. [13]. For any stochastic matrix $M$ in canonical form with $m$ ergodic classes, as in (2.1), there exist $l_{1}, \ldots, l_{m} \in \mathbb{N} \backslash\{0\}$ such that every $l_{j}$ does not exceed the order of $M_{j}$, and $\lim _{q \rightarrow \infty}\left(M_{j}{ }^{l_{j}}\right)^{q}$ exists. Moreover, $\lim _{q \rightarrow \infty}\left(T_{m+1}\right)^{q}=0$.

Actually, every $l_{j}$ in Theorem 2.3 coincides with the period of the ergodic class represented by $M_{j}$ [13]. The following lemma describes the powers of a square stochastic matrix in canonical form:

Lemma 2.4. Let $M$ be a stochastic matrix in canonical form as in $(2.1)$, with $l_{j}$ being the period of the ergodic class represented by $M_{j}$, for every $1 \leq j \leq m$. Given $h \in \mathbb{N}$, we have

$$
M^{h}=\left[\begin{array}{ccccc}
M_{1}^{h} & 0 & \cdots & 0 & 0 \\
\vdots & & \ddots & & \vdots \\
0 & 0 & \cdots & M_{m}^{h} & 0 \\
\hat{T}_{1, h} & \hat{T}_{2, h} & \cdots & \hat{T}_{m, h} & T_{m+1}^{h}
\end{array}\right]
$$

for suitable matrices $\hat{T}_{1, h}, \ldots, \hat{T}_{m, h}$. Moreover, for every $1 \leq j \leq m$ and every $0 \leq r_{j}<l_{j}, \lim _{q \rightarrow \infty} \hat{T}_{j, q l_{j}+r_{j}}$ exists.

A language $L$ is said to be unary whenever it is defined over a single-letter alphabet. In this case, we let $L \subseteq a^{*}$. A unary language $L$ is cyclic (or periodic) if there exists $d \in \mathbb{N} \backslash\{0\}$ such that, for any $h \in \mathbb{N}$, we have $a^{h} \in L$ if and only if $a^{h+d} \in L$. In this case, we say that $L$ is $d$-cyclic. Moreover, if $\nexists d^{\prime}<d$ such that $L$ is $d^{\prime}$-cyclic, then we say that $L$ is properly $d$-cyclic.

We assume that the reader is familiar with the notion of deterministic and nondeterministic finite automata. We briefly recall the notion of probabilistic finite automata, already restricted to the unary case. 
A unary PFA with set of states $\left\{q_{1}, q_{2}, \ldots, q_{s}\right\}$ is described by a triple $A=$ $(\pi, M, \eta)$, where $\pi \in[0,1]^{s}$ is a stochastic row vector representing the probability distribution of the initial state, $M$ is a stochastic transition matrix of order $s$, where $(M)_{i, j}$ is the probability that $A$ moves from state $q_{i}$ to state $q_{j}$ upon reading $a$, $\eta \in\{0,1\}^{s}$ is the characteristic column vector of the set of final states.

The probability that $A$ accepts the input string $a^{h}$ is defined as $p_{A}\left(a^{h}\right)=\pi M^{h} \eta$. Given a real number $\lambda, 0 \leq \lambda<1$, the language accepted by $A$ with cut-point $\lambda$ is the set $L_{A, \lambda}=\left\{a^{h} \mid h \in \mathbb{N}\right.$ and $\left.p_{A}\left(a^{h}\right)>\lambda\right\}$. The cut-point $\lambda$ is said to be isolated if there exists $\delta>0$ such that $p_{A}\left(a^{h}\right) \geq \lambda+\delta$ for each $a^{h} \in L$, and $p_{A}\left(a^{h}\right) \leq \lambda-\delta$ for each $a^{h} \notin L$. In this case, $\lambda$ is also said to be $\delta$-isolated.

From the results of Rabin [12], it is well-known that the class of languages accepted by PFAs with isolated cut-points coincides with the class of regular languages, and, on the other hand, nonregular languages can be accepted (even in the unary case), with nonisolated cut-points (see also [11]).

We will always assume our automata to be in canonical form, so, provided the transition matrix $M$ has $m$ ergodic components, it is in the form given in (2.1). Moreover, the vectors $\pi$ and $\eta$ can be written as $\pi=\bigoplus_{j=1}^{m+1} \pi_{j}$ and $\eta=\bigoplus_{j=1}^{m+1} \eta_{j}$ according to the same state partition. The states associated to the ergodic components will be called ergodic states, while the remaining will be called transient states. Given a PFA $A$ with an isolated cut point $\lambda$, we say that another PFA $A^{\prime}$ is equivalent to $A$ if there exists a value $\lambda^{\prime}$, which is isolated for $A^{\prime}$, such that $L_{A, \lambda}=L_{A^{\prime}, \lambda^{\prime}}$. Notice that the isolation of the two cut points may be different.

\section{Chrobak normal Form}

A NFA is in Chrobak normal form [2] if it consists of an initial deterministic path, ending in a state connected to a set of disjoint deterministic cycles, via nondeterministic transitions.

In terms of matrix representation, a NFA in Chrobak normal form can be described, according to (2.1) as follows:

- each $M_{j} \in\{0,1\}^{t_{j} \times t_{j}}$ defines a deterministic cycle of length $t_{j}$, which means

$$
\left(M_{j}\right)_{i, k}= \begin{cases}1 & \text { if } k \equiv i+1 \quad\left(\bmod t_{j}\right) \\ 0 & \text { otherwise }\end{cases}
$$

- $T_{m+1} \in\{0,1\}^{t_{m+1} \times t_{m+1}}$ describes the initial path of length $t_{m+1}$, so we have

$$
\left(T_{m+1}\right)_{i, k}= \begin{cases}1 & \text { if } k=i+1 \\ 0 & \text { otherwise }\end{cases}
$$

- for each $1 \leq j \leq m, T_{j} \in\{0,1\}^{t_{m+1} \times t_{j}}$ describes the nondeterministic connection between the last state of the path and one state of the $j$ th cycle, more formally

$$
\left(T_{j}\right)_{i, k}= \begin{cases}1 & \text { if }(i, k)=\left(t_{m+1}, 1\right) \\ 0 & \text { otherwise }\end{cases}
$$




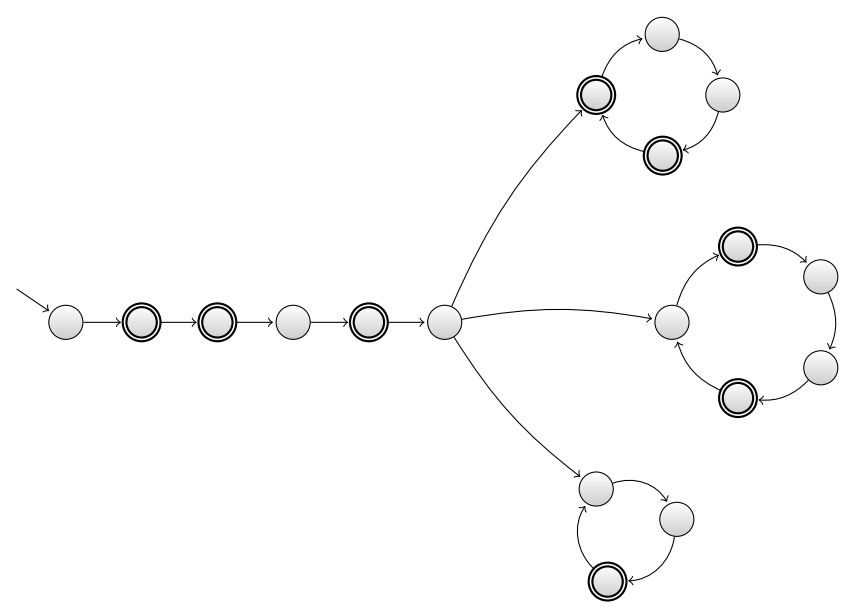

Figure 1. Example of NFA in Chrobak normal form.

The initial vector is such that $\pi_{j}=0$ for each $1 \leq j \leq m$ and $\pi_{m+1}=e_{m+1}$. The final vector $\eta$ can be any boolean vector, which means that in each cycle there can be more than one accepting state. An example of NFA in Chrobak normal form is given in Figure 1. ${ }^{2}$

It is shown in [2] that each unary $n$-state NFA can be turned into an equivalent NFA in Chrobak normal form with at most $n$ states in the cycles and $O\left(n^{2}\right)$ states in the initial path (see [3] for a finer estimation).

In Chrobak normal form, the only nondeterministic decision is taken in the last state of the initial path, by choosing one of the transitions leading to the cycles. Notice that, for each cycle, there is exactly one transition from the last state of the path to one state in the cycle; such a state can be considered the "initial state of the cycle".

To define a probabilistic version of Chrobak normal form, it seems to be natural to replace the only possible nondeterministic choice by a probabilistic choice. In other words, from the last state of the initial path, the automaton chooses one of the possible cycles (and thus the corresponding initial state) according to a probabilistic distribution. In the above matrix representation, the only change concerns matrices $T_{j}$, for $1 \leq j \leq m$, which have to satisfy the following conditions:

$$
\left(T_{j}\right)_{i, k}\left\{\begin{array}{ll}
\in[0,1] & \text { if }(i, k)=\left(t_{m+1}, 1\right) \\
=0 & \text { otherwise, }
\end{array} \text { and } \sum_{j=1}^{m}\left(T_{j}\right)_{t_{m+1}, 1}=1 .\right.
$$

We consider the problem of converting a PFA in Chrobak normal form into an equivalent DFA.

\footnotetext{
${ }^{2}$ Throughout the paper, we will depict unary NFAs using transitions graphs. The accepting states will be indicated with a double circle. The initial state(s) will be marked with an entering edge.
} 
Theorem 3.1. For each unary n-state PFA $M$ in Chrobak normal form, there exists an equivalent DFA $M^{\prime}$ with $\mathrm{e}^{O(\sqrt{n \cdot \ln n})}$ states. Furthermore, the number of states in the initial path of $M^{\prime}$ is the same as in the initial path of $M$ (besides a possible dead state, if $M$ does not have any cycle).

Proof. Essentially, it is possible to apply the same construction given in [2] for the conversion from NFAs in Chrobak normal form into DFAs: the initial path of the resulting automaton coincides with that of the given automaton, the cycle simulates "in parallel" all the cycles of the given automaton. To do this, it is enough to take, as length of the cycle, the least common multiple of cycle lengths in the given automaton. The only difference is in the choice of final states in the cycle. Each state of $M^{\prime}$ represents a tuple of states of $M$ (one for each cycle in the original automaton). In order to make final a state of $M^{\prime}$, in the nondeterministic case it is enough to have a final state of $M$ in the corresponding tuple. In the probabilistic case, we have to calculate the sum of the probabilities of entering the cycles whose states in the tuple are final. The state of $M^{\prime}$ under consideration is final if and only if such a sum exceeds the cut-point.

The bound on the number of the states immediately derives from the results of Chrobak.

We will show now that with this definition we cannot get descriptional complexity properties similar to those for NFAs in Chrobak normal form. In fact, this form does not match neither the bound on the number of states in the initial path (i.e., the noncyclic part of the language) nor that on the number of states in the cycles.

Let us start by considering the initial path. For each integer $k>0$ we consider the following language

$$
L_{k}=\left\{a^{h} \mid h \leq 2 k\right\} \cup\left\{a^{2 h+1} \mid h \geq k\right\} .
$$

Clearly $L_{k}$ can be recognized by the following 4 -state pfa $^{3}$

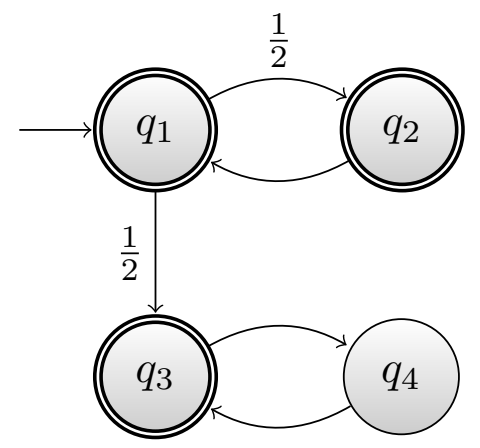

\footnotetext{
${ }^{3}$ As for NFAs, we will depict also unary PFAs using transitions graphs. The probability of a transition will be represented by a label on the corresponding edge. Possible initial states, i.e., states with a nonnull probability to be chosen at the beginning of the computation, will be marked with an entering edge, labeled with the corresponding probability. Usually, we will omit probabilities equal to 1 .
} 
with cut-point $\frac{1}{2^{k}}-\frac{1}{2^{k+2}}$ isolated by $\frac{1}{2^{k+2}}$. On the other hand, it is not difficult to verify that each DFA accepting it must have an initial path of at least $2 k$ states. This allows us to state the following:

Theorem 3.2. For every $n \in \mathbb{N}$, there exists a language $L_{n}$ such that

- $L_{n}$ is accepted by a PFA with 4 states;

- if a DFA made of an initial path of $t$ states and a cycle of $p$ states recognizes $L_{n}$, then it must hold $t \geq n$.

In the light of Theorem 3.1, this implies also that each PFA for $L_{n}$ in Chrobak normal form should have an initial path of at least $n$ states. Hence, we have proved the following:

Corollary 3.3. For all integers $n$ there exists a language $L_{n}$ which is accepted by a PFA with 4 states, but requires more than $n$ states to be accepted by a PFA in Chrobak normal form.

\section{Normal Form For PFAS ACCEPTING CYCLIC LANGUAGES}

Now, we move our attention to the cyclic part of automata and languages. We will show that, even to express this part, PFAs in Chrobak normal form can be bigger than PFAs. This result will be stated considering cyclic languages.

We recall that, as observed in [6], each NFA in Chrobak normal form accepting a cyclic language can be reduced to one of the following two forms:

(a) one deterministic cycle (namely, the initial path is empty and, actually, the automaton is deterministic);

(b) an initial state with outgoing nondeterministic transitions connecting to some disjoint cycles (namely, the initial path is reduced to the initial state).

It easy to observe that, by allowing multiple initial states, the form (b) can be further simplified, reducing the automaton to a collection of disjoint cycles, each of them having one initial state. At the beginning of the computation one cycle (i.e., its initial state) is nondeterministically chosen. Hence, form (a) can be seen as a special case, with only one loop.

We can do the same in the probabilistic case, reducing the Chrobak normal form for PFAs accepting unary languages to a collection of disjoint cycles, each one having exactly one initial state. At the beginning of the computation, a probabilistic distribution is used to select one among these possible initial states.

In the following, the next lemma will be useful:

Lemma 4.1. Let $\mathcal{L}$ be a properly $d$-cyclic language accepted by a PFA with an isolated cut-point and $m$ ergodic components of periods $l_{1}, \ldots, l_{m}$. If, according to the Fundamental Theorem of Arithmetic, $d$ factorizes as $p_{1}^{k_{1}} p_{2}^{k_{2}} \cdots p_{n}^{k_{n}}$, then for each $1 \leq i \leq n$ there exists $1 \leq j \leq m$ such that $p_{i}^{k_{i}}$ divides $l_{j}$. 
Proof. By [9], Theorem 2.7, we know that $\mathcal{L}$ must be $\ell$-cyclic, for $\ell=$ $\operatorname{lcm}\left(l_{1}, \ldots l_{m}\right)$. Indeed, $\mathcal{L}$ being properly $d$-cyclic, $d$ must divide $\ell$. Hence, for some integer $\kappa \geq 0$, we have that

$$
\kappa d=\ell=\operatorname{lcm}\left(l_{1}, \ldots, l_{m}\right)
$$

Now, for a suitable $s \geq n$, let $\prod_{t=1}^{s} p_{t}^{\gamma_{t}}$ be the prime factorization of $\ell$ where, as usual, we let $\gamma_{t}=\max \left\{\alpha \in \mathbb{N} \mid p_{t}^{\alpha}\right.$ divides $l_{j}$ for $\left.1 \leq j \leq m\right\}$. Equality (4.1) can thus be rewritten as

$$
\kappa \prod_{i=1}^{n} p_{i}^{k_{i}}=\prod_{t=1}^{s} p_{t}^{\gamma_{t}} .
$$

This clearly shows that, for each $1 \leq i \leq n$ there must exist $1 \leq t \leq s$ such that $p_{i}{ }^{k_{i}}$ divides $p_{t}^{\gamma_{t}}$. In turn, by definition of least common multiple, we have that $p_{t}^{\gamma_{t}}$ must divide $l_{r}$, for some $1 \leq r \leq m$, whence the result follows.

We are now able to prove that there are languages for which PFAs in Chrobak normal form cannot be as small as PFAs:

Theorem 4.2. There exist infinitely many cyclic languages accepted by PFAs smaller than each equivalent PFA in Chrobak normal form.

Proof. Given two primes $p<p^{\prime}$, with $p, p^{\prime} \geq 2$, consider the language

$$
L_{p, p^{\prime}}=\left(a^{p \cdot p^{\prime}}\right)^{*}+a\left(a^{p^{\prime}}\right)^{*} .
$$

We can verify that $L_{p, p^{\prime}}$ is accepted with isolated cut-point $\frac{3}{8}$ by the following PFA $M$ :
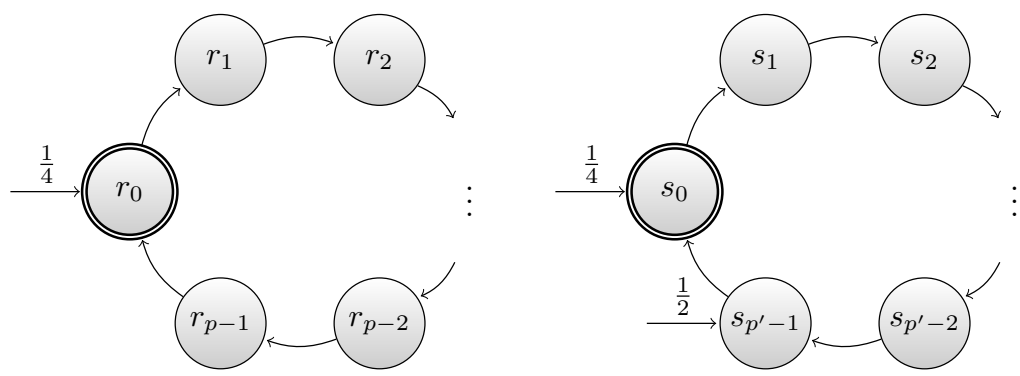

By Lemma 4.1, this implies that each PFA for $L_{p, p^{\prime}}$ must have either at least two ergodic components of periods multiple of $p$ and $p^{\prime}$ respectively, or at least one ergodic component of period multiple of $p \cdot p^{\prime}$. Let $A$ be a PFA in Chrobak normal form for $L_{p, p^{\prime}}$. Since $p \cdot p^{\prime}>p+p^{\prime}$ for primes $p^{\prime}>p \geq 2$ we are considering, the only possibility to have $A$ not bigger than $M$ is that $A$ is isomorphic to the 
following automaton:
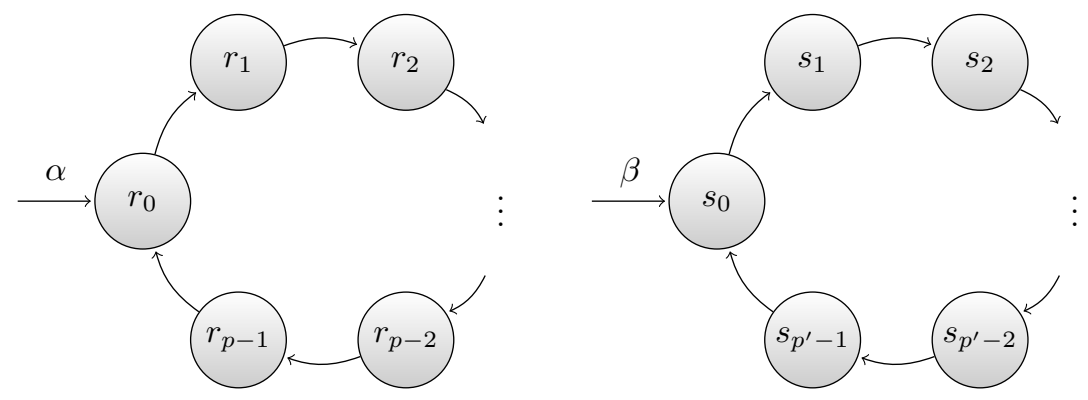

for a suitable choice of $\alpha, \beta$ (i.e., the initial vector $\mu$ ) and accepting states (i.e., the final vector $\eta$ ). We define variables $\alpha_{i}, \beta_{j}$, for $i \in\{0,1, \ldots, p-1\}, j \in$ $\left\{0,1, \ldots, p^{\prime}-1\right\}$ as follows:

$$
\alpha_{i}=\left\{\begin{array}{ll}
\alpha & \text { if } r_{i} \text { is accepting } \\
0 & \text { otherwise }
\end{array} \quad \beta_{j}= \begin{cases}\beta & \text { if } s_{j} \text { is accepting } \\
0 & \text { otherwise }\end{cases}\right.
$$

The probability of accepting a word $a^{h}$ on $A$ is exactly $\alpha_{h \bmod p}+\beta_{h \bmod p^{\prime}}$. In particular, considering the definition of the language, we get the following inequalities:

$$
\begin{array}{ll}
\text { from } \varepsilon \in L_{p, p^{\prime}}: & \alpha_{0}+\beta_{0}>\lambda \\
\text { from } a^{p} \notin L_{p, p^{\prime}}: & \alpha_{0}+\beta_{p \bmod p^{\prime}}<\lambda \\
\text { from } a^{p^{\prime}} \notin L_{p, p^{\prime}}: & \alpha_{p^{\prime} \bmod p}+\beta_{0}<\lambda \\
\text { from } a^{k p^{\prime}+1} \in L_{p, p^{\prime}}, \text { for all } k \in \mathbb{N}: & \alpha_{\left(k p^{\prime}+1\right) \bmod p}+\beta_{1}>\lambda .
\end{array}
$$

From the first three inequalities we get that $\alpha_{0}>\alpha_{p^{\prime} \bmod p}$, and $\beta_{0}>\beta_{p \bmod p^{\prime}}$, therefore both $r_{0}$ and $s_{0}$ must be accepting, and $\alpha_{0}=\alpha, \beta_{0}=\beta$, while $\alpha_{p^{\prime} \bmod p}=$ $\beta_{p \text { mod } p^{\prime}}=0$. Because of (4.5) and (4.6), both $\alpha_{0}$ and $\beta_{0}$ cannot reach $\lambda$ on their own, so we have $\alpha, \beta<\lambda$. This implies that in (4.7), for each value of $k$, neither $\beta_{1}$ nor $\alpha_{\left(k p^{\prime}+1\right) \bmod p}$ can be zero. Since $p$ and $p^{\prime}$ are coprime, it holds $\left\{\left(k p^{\prime}+1\right) \bmod p \mid k \in \mathbb{N}\right\}=\{0,1, \ldots, p\}$, so all states $r_{0}, r_{1}, \ldots, r_{p-1}$ must be accepting, which contradicts $\alpha_{p^{\prime} \bmod p}=0$.

Theorem 4.2 shows that the conversion of PFAs into Chrobak normal form requires in general an increase in the number of states. To overcome this problem, we here define a new normal form for PFAs recognizing periodic languages, called cyclic normal form. We will prove that for any unary PFA accepting a periodic language there exists an equivalent PFA in cyclic normal form with at most the same number of states. As a consequence, each periodic language admits a minimal PFA in cyclic normal form. 
Definition 4.3. A unary PFA $A=(\pi, M, \eta)$ with $s$ states accepting a cyclic language is in cyclic normal form if there exist $l_{1}, \ldots, l_{m} \in \mathbb{N}$ such that

- $\sum_{j=1}^{m} l_{j}=s$;

- $\pi=\bigoplus_{j=1}^{m} \pi_{j}$, where $\pi_{j} \in \mathbb{R}^{1 \times l_{j}}$ and $\sum_{i=1}^{s}(\pi)_{i}=1$;

- $M=\bigoplus_{j=1}^{m} M_{j}$, where $M_{j} \in \mathbb{R}^{l_{j} \times l_{j}}$ and $M_{j}=\left[\begin{array}{cccc}0 & \cdots & 0 & 1 \\ 1 & \cdots & 0 & 0 \\ \vdots & \ddots & \vdots & \vdots \\ 0 & \cdots & 1 & 0\end{array}\right]$;

- $\eta=\bigoplus_{j=1}^{m} \eta_{j}$, where $\eta_{j} \in \mathbb{R}^{l_{j} \times 1}$ and $\eta_{j}=e_{1}^{T}$.

More intuitively, $A$ is a collection of disjoint deterministic cycles of lengths $l_{1}, \ldots, l_{m}$, each of them having a unique final state (vectors $\eta_{j}$ ) and more than one initial state (vectors $\pi_{j}$ ). An example of PFA in strictly normal form is the automaton $M$ given in the proof of Theorem 4.2 .

Now, we are able to prove our main result:

Theorem 4.4. For each unary PFA $A$ accepting a cyclic language, there exists an equivalent PFA $A^{\prime}$ in cyclic normal form with at most the same number of states.

Proof. Let $L$ be the language accepted by $A$, and $d$ an integer such that $L$ is $d$-cyclic. If $L=\Sigma^{*}$ or $L=\emptyset$, then it admits a trivial PFA in cyclic normal form, so we prove the theorem for $L \neq \Sigma^{*}$ and $L \neq \emptyset$.

Suppose that $A=(\pi, M, \eta)$ recognizes the language $L$ with a $\delta$-isolated cutpoint $\lambda$. Assume $A$ is in canonical form with $m$ ergodic components of periods $l_{1}, \ldots, l_{m}$, so the matrix describing the transitions of $A$ in $h$ steps has the form given in (2.2). It is not hard to see that the acceptance probability of a word $a^{h}$ is

$$
\pi M^{h} \eta=\sum_{j=1}^{m}\left(\pi_{j} M_{j}^{h} \eta_{j}+\pi_{m+1} \hat{T}_{j, h} \eta_{j}\right)+\pi_{m+1} T_{m+1}^{h} \eta_{m+1} .
$$

Since $\lim _{h \rightarrow \infty} \pi_{m+1} T_{m+1}^{h} \eta_{m+1}=0$, by calling $\tilde{p}(j, h)=\pi_{j} M_{j}^{h} \eta_{j}+\pi_{m+1} \hat{T}_{j, h} \eta_{j}$, we can write

$$
\left|\pi M^{h} \eta-\sum_{j=1}^{m} \tilde{p}(j, h)\right|<\varepsilon(h)
$$

for some decreasing function $\varepsilon$ which tends to zero. Because of (4.8) and Lemma 2.4, we can find a value $H>0$ such that for each $1 \leq j \leq m$ it holds

$$
\begin{array}{r}
\left|\pi M^{H d} \eta-\sum_{j=1}^{m} \tilde{p}(j, H d)\right|<\frac{\delta}{4}, \\
\forall i, k \geq 0:\left|\tilde{p}(j, H d+i)-\tilde{p}\left(j, H d+k l_{j}+i\right)\right|<\frac{\delta}{4 m} .
\end{array}
$$


Let us now define, for $1 \leq j \leq m$ and $0 \leq r_{j} \leq l_{j}$, the probability distribution

$$
p\left(j, r_{j}\right)=\frac{\tilde{p}\left(j, H d+r_{j}\right)}{\sum_{\gamma=1}^{m} \sum_{i=1}^{l_{\gamma}} \tilde{p}(\gamma, H d+i)} .
$$

Let $A^{\prime}=\left(\pi^{\prime}, M^{\prime}, \eta^{\prime}\right)$, where $M^{\prime}$ and $\eta^{\prime}$ have the form described in Definition 4.3 and

$$
\pi^{\prime}=\bigoplus_{j=1}^{m}\left(p(j, 0), p(j, 1), \ldots, p\left(j, l_{j}-1\right)\right)
$$

The event induced by $A^{\prime}$ is

$$
\pi^{\prime}\left(M^{\prime}\right)^{h} \eta^{\prime}=\sum_{j=1}^{m} p\left(j, h \bmod l_{j}\right)
$$

By defining

$$
\lambda^{\prime}=\frac{\lambda}{\sum_{\gamma=1}^{m} \sum_{i=1}^{l_{\gamma}} \tilde{p}(\gamma, H d+i)} \quad \text { and } \quad \delta^{\prime}=\frac{\delta}{2 \sum_{\gamma=1}^{m} \sum_{i=1}^{l_{\gamma}} \tilde{p}(\gamma, H d+i)}
$$

and by applying (4.9)-(4.12), we get the following implications:

$$
\begin{aligned}
a^{h} \in L & \Rightarrow a^{H d+h} \in L \\
& \Rightarrow \pi M^{H d+h} \eta \geq \lambda+\delta \\
& \Rightarrow \sum_{j=1}^{m} \tilde{p}(j, H d+h) \geq \lambda+\frac{3 \delta}{4} \\
& \Rightarrow \sum_{j=1}^{m} \tilde{p}\left(j, H d+h \bmod l_{j}\right) \geq \lambda+\frac{\delta}{2} \\
& \Rightarrow \sum_{\gamma=1}^{m} \sum_{i=1}^{l_{\gamma}} \tilde{p}(\gamma, H d+i) \sum_{j=1}^{m} p\left(j, h \bmod l_{j}\right) \geq \lambda+\frac{\delta}{2} \\
& \Rightarrow \sum_{j=1}^{m} p\left(j, h \bmod l_{j}\right) \geq \lambda^{\prime}+\delta^{\prime} \\
& \Rightarrow \pi^{\prime}\left(M^{\prime}\right)^{h} \eta^{\prime} \geq \lambda^{\prime}+\delta^{\prime},
\end{aligned}
$$


and:

$$
\begin{aligned}
a^{h} \notin L & \Rightarrow a^{H d+h} \notin L \\
& \Rightarrow \pi M^{H d+h} \eta \leq \lambda-\delta \\
& \Rightarrow \sum_{j=1}^{m} \tilde{p}(j, H d+h) \leq \lambda-\frac{3 \delta}{4} \\
& \Rightarrow \sum_{j=1}^{m} \tilde{p}\left(j, H d+h \bmod l_{j}\right) \leq \lambda-\frac{\delta}{2} \\
& \Rightarrow \sum_{\gamma=1}^{m} \sum_{i=1}^{l_{\gamma}} \tilde{p}(\gamma, H d+i) \sum_{j=1}^{m} p\left(j, h \bmod l_{j}\right) \leq \lambda-\frac{\delta}{2} \\
& \Rightarrow \sum_{j=1}^{m} p\left(j, h \bmod l_{j}\right) \leq \lambda^{\prime}-\delta^{\prime} \\
& \Rightarrow \pi^{\prime}\left(M^{\prime}\right)^{h} \eta^{\prime} \leq \lambda^{\prime}-\delta^{\prime} .
\end{aligned}
$$

Equations (4.13) and (4.14), together with the fact that $L$ is neither empty nor the whole $\Sigma^{*}$, imply

$$
\lambda^{\prime}+\delta^{\prime} \leq 1 \quad \text { and } \quad \lambda^{\prime}-\delta^{\prime} \geq 0
$$

therefore $A^{\prime}$ recognizes $L$ with cut-point $\lambda^{\prime}$ isolated by $\delta^{\prime}$.

Notice that, by exploiting the structure of the cyclic normal form, we can determine an upper bound (other than the trivial minimum DFA) on the size of the minimal PFA in Chrobak normal form: in fact, to convert any PFA from cyclic to Chrobak form, it is sufficient to replace each cycle with initial states $q_{1}, q_{2}, \ldots, q_{k}$, with $k$ copies of that cycle, where the $i$ th copy has as unique initial state the copy of $q_{i}$.

Finally, we remark that a cyclic normal form can be defined also on NFAs by requiring $\pi$ to be a boolean vector. By allowing multiple initial states, as we did for the Chrobak normal form for NFAs accepting cyclic languages, the conversion into cyclic normal form does not increase the number of states.

\section{Normal FORM FOR PFAS ACCEPting NON CYCLIC LANGUAGES}

We now extend the definition of cyclic normal form in order to accept also regular languages which are non periodic. This is done by adding an initial path of states.

In this way, a NFA in cyclic normal form is similar to one in Chrobak normal form: there is an initial deterministic path and a set of disjoint deterministic cycles. 


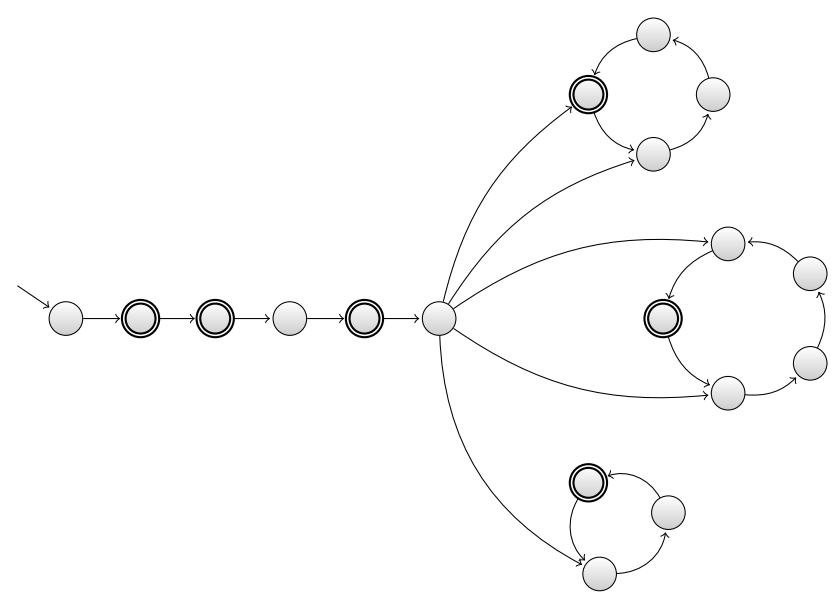

FiguRE 2. NFA in cyclic normal form equivalent to the one in Chrobak normal form of Figure 1.

The differences concern the final states and the nondeterministic transitions: each cycle must contain exactly one final state, however, from the last state in the initial path, many different states, even belonging to a same loop, can be reached in a nondeterministic way. Even in this form, the only nondeterministic choice is taken in the last state of the initial path.

An easy construction can be done to transform each NFA in Chrobak normal form into an equivalent NFA in cyclic normal form and viceversa, by keeping the same initial path and the same set of cycles. Hence, for NFAs these two forms can be considered equivalent, even in terms of the number of states (an example of NFA converted from Chrobak into cyclic normal form is given in Fig. 2).

The probabilistic version of cyclic normal form is defined by replacing the only nondeterministic choice by a probabilistic distribution, on all the states in the cycles, namely, from the last state of the initial path, the automaton chooses one state in the cycles, according to such a distribution. In the matrix representation, matrices $M_{j}$ and $T_{m+1}$ are as in Chrobak normal form, while for matrices $T_{j}$, $j=1, \ldots, m$, the following conditions must be satisfied:

$$
\left(T_{j}\right)_{i, k}\left\{\begin{array}{ll}
\in[0,1] & \text { if } i=t_{m+1} \\
=0 & \text { otherwise, }
\end{array} \text { and } \sum_{j=1}^{m} \sum_{k=1}^{t_{j}}\left(T_{j}\right)_{t_{m+1}, k}=1\right.
$$

Furthermore, the vector of final states $\eta$ can be decomposed in $m+1$ vectors $\eta_{1}, \eta_{2}, \ldots, \eta_{m}, \eta_{m+1}$, where, for $i=1, \ldots, m$, the vector $\eta_{i}$ corresponding to $i$ th cycle has exactly one component equal to 1. 
Refining the argument used in the proof, we can adapt Theorem 3.1 to the conversion of PFAs in cyclic normal form into DFAs:

Theorem 5.1. For each unary n-state PFA $M$ in cyclic normal form, there exists an equivalent DFA $M^{\prime}$ with $e^{O(\sqrt{n \cdot \ln n})}$ states. Furthermore, the number of states in the initial path of $M^{\prime}$ is the same as in the initial path of $M$ (besides a possible dead state, if $M$ does not have any cycle).

Finally, we analyze the cost of converting a PFA into cyclic normal form: Theorems 5.1 and 3.2 imply that this transformation generates an arbitrarily long initial path, like in the Chrobak normal form. However, Theorem 4.4 guarantees that the number of states involved in the cyclic part of the language is not increased after the conversion. By summarizing, in the non periodic case Theorems 4.2 and 4.4 generalize to the following

Corollary 5.2. There exist infinitely many unary regular languages recognized by PFAs with less ergodic states than the ergodic states of any equivalent PFA in Chrobak normal form.

Corollary 5.3. For each unary language $L$ recognized by a minimal PFA $A$, there exists a PFA $A^{\prime}$ in cyclic normal form recognizing $L$ with as many ergodic states as $A$.

\section{Conclusions And open problems}

In this paper we have studied the natural extension of Chrobak normal form to the probabilistic framework: in Theorem 4.2 we have shown that the conversion of a PFA into Chrobak normal form requires an increase of both transient and ergodic states. We then proposed an alternative normal form and, in Theorem 4.4, we have shown that converting a PFA into cyclic normal form does not require an increment of states in the ergodic component, although the length of the initial path cannot be bounded in terms of the size of the minimal PFA. It would be interesting to study whether it is possible to bound this length in terms of other parameters like, for example, the isolation of the cut point.

The advantage of the cyclic normal form is that it guarantees a simple and compact structure for minimal PFAs, where probabilism is restricted to a single step of computation and the acceptance probability of the input words can be easily obtained by looking at one single transition for each cycle. On the other hand, this form does not preserve the original cut point nor the isolation: in fact, consider a prime $p>2$ and the language $L_{p}=\left\{a^{h} \mid h \bmod p \neq 0\right\}$. The minimal DFA for $L_{p}$ is made of a single cycle of length $p$, with $p-1$ accepting states, and it is also a PFA in Chrobak normal form accepting $L_{p}$ exactly. However, the equivalent PFA in cyclic normal form, obtained with the conversion given in the proof of Theorem 4.4 , has cut point $1 /(p-1)$ isolated by $1 /(2(p-1))$. The aim of this paper was to provide the simplest normal form for PFAs which guarantees 
an isolated cut point and minimality in the number of states. If the interest is to preserve recognition with high probability, one could consider relaxations of this normal form, for example by allowing multiple accepting states in each cycle.

Another interesting problem is the extension of this normal form to the two-way probabilistic model. Since two-way unary PFAs with isolated cut point still recognize only regular languages (as shown in [7]), having a normal form similar to the Chrobak or cyclic one could be helpful to obtain state bounds on the conversion between unary two-way PFAs and one-way DFAs.

Acknowledgements. The authors would like to thank the reviewers for their careful work and stimulating comments. In particular, the example of the language $L_{p}$ presented in the Conclusion was suggested by a referee.

\section{REFERENCES}

[1] M.P. Bianchi, C. Mereghetti, B. Palano and G. Pighizzini, On the size of unary probabilistic and nondeterministic automata. Fundamenta Informaticae 112 (2011) 119-135.

[2] M. Chrobak, Finite automata and unary languages, Theoret. Comput. Sci. 47 (1986) 149-158; Erratum, Theoret. Comput. 302 (2003) 497-498.

[3] V. Geffert, Magic numbers in the state hierarchy of finite automata. Inf. Comput. 205 (2001) 1652-1670.

[4] G. Gramlich, Probabilistic and Nondeterministic Unary Automata, in Proc. of MFCS. Lect. Notes Comput. Sci. 2757 (2003) 460-469.

[5] D.L. Isaacson and R.W. Madsen, Markov Chains Theory and Applications, edited by J. Wiley \& Sons, Inc. (1976).

[6] T. Jiang, E. Mcdowell and B. Ravikumar, The structure and complexity of minimal NFA's over a unary alphabet. Int. J. Found. Comput. Sci. 2 (1991) 163-182.

[7] J. Kaneps, Regularity of One-Letter Languages Acceptable by 2-Way Finite Probabilistic Automata, in Proc of FCT. Lect. Notes Comput. Sci. 529 (1991) 287-296.

[8] C. Mereghetti and G. Pighizzini, Optimal simulations between unary automata. SIAM J. Comput. 30 (2001) 1976-1992.

[9] C. Mereghetti, B. Palano and G. Pighizzini, Note on the succinctness of deterministic, nondeterministic, probabilistic and quantum finite automata. RAIRO-Theor. Inf. Appl. $\mathbf{5}$ (2001) 477-490.

[10] M. Milani and G. Pighizzini, Tight bounds on the simulation of unary probabilistic automata by deterministic automata. J. Automata, Languages and Combinatorics 6 (2001) 481-492.

[11] A. Paz, Introduction to Probabilistic Automata. Academic Press, New York (1971).

[12] M. Rabin, Probabilistic automata. Inf. Control 6 (1963) 230-245.

[13] E. Seneta, Non-negative Matrices and Markov Chains, 2nd edition. Springer-Verlag (1981).

[14] A.W. To, Unary finite automata vs. arithmetic progressions. Inf. Process. Lett. 109 (2009) $1010-1014$.

Communicated by C. Mereghetti.

Received November 4, 2011. Accepted June 8, 2012. 